\title{
Dynamic Decomposition of Motion in Essential and Parkinsonian Tremor
}

\author{
Fariborz Rahimi, Derek Debicki, Angela Roberts-South, Carina Bee, Priya Bapat, \\ Mandar Jog
}

\begin{abstract}
Background: Treatment options for essential (ET) and Parkinson disease (PD) tremor are suboptimal, with significant side effects. Botulinum toxin type A (BoNT A) is successfully used in management of various focal movement disorders but is not widely used for tremor. Method: This study examines complexity of wrist tremor in terms of involvement of its three anatomical degrees of freedom (DOF) in two common situations of rest and posture. The study examines tremor in 11 ET and 17 PD participants by kinematic decomposition of motion in 3-DOF. Results: Tremor decomposition showed the motion involved more than one DOF ( $<70 \%$ contribution in one DOF) in most ET (rest: 100\%, posture: 64\%) and PD (rest: 77\%, posture: 77\%) patients. Task variation resulted in change in both amplitude and composition in ET, but not in PD. Amplitude significantly increased from rest to posture in ET. Directional bias was observed at the wrist for ET (pronation), and PD (extension, ulnar deviation, pronation). Average agreement between clinical visual and kinematic selection of muscles was $55 \%$ across all subjects. Conclusion: This study shows the complexity of tremor and the difficulty in visual judgment of tremor, which may be key to the success of targeted focal treatments such as BoNT A.
\end{abstract}

RÉSUMÉ: Décomposition dynamique du mouvement dans le tremblement essential et le tremblement parkinsonien. Contexte : Les options de traitement du tremblement essentiel (TE) et du tremblement dû à la maladie de Parkinson (MP) sont sous-optimales et comportent des effets secondaires importants. La toxine botulique de type A (BoNT A) est utilisée avec succès dans le traitement de différents troubles focaux du mouvement, mais elle n'est pas utilisée couramment pour traiter le tremblement. Méthode : Cette étude examine la complexité du tremblement du poignet en termes de ses trois degrés anatomiques de liberté (DDL) dans deux situations fréquentes soit le tremblement de repos et le tremblement postural. L'étude examine le tremblement chez 11 patients atteints de TE et 17 patients atteints de MP au moyen de la décomposition cinématique du mouvement en 3 DDL. Résultats: La décomposition du tremblement a montré que le mouvement impliquait plus d'un DDL (contribution $<70 \%$ dans un DDL) chez la plupart des patients atteints de TE (de repos : 100\%; postural : 64\%) et chez les patients atteints de MP (de repos : 77\% ; postural : 77\%). Une variation des tâches provoquait un changement tant de l'amplitude que de la composition dans le TE, mais non dans la MP. L'amplitude augmentait significativement du repos à la situation du maintien d'une posture dans le TE. Un biais directionnel a été observé au niveau du poignet dans le TE (pronation) et dans la MP (extension, déviation cubitale, pronation). La concordance moyenne entre la sélection visuelle clinique et cinématique des muscles était de 55\% pour l'ensemble des sujets. Conclusion : Cette étude montre la complexité du tremblement et la difficulté de porter un jugement visuel sur le tremblement, un élément clé du succès de traitements focaux ciblés tels la BoNT A.

Keywords: Essential tremor, Parkinsonian tremor, botulinum toxin type A, kinematics, tremor composition doi:10.1017/cjn.2015.12

Can J Neurol Sci. 2015; 42: 116-124

Tremor is one of the most prevalent adult movement disorders. ${ }^{1}$ Many muscles are involved with the motion required to generate tremor, particularly in the upper limb. However, to date, the complexity and variability of tremor movements at a particular joint have not yet been effectively explored, characterized, and decomposed into the constituent separate motions such as flexion/ extension, pronation/supination etc. Although visual evaluation of tremor by a physician is often sufficient in order to make clinical diagnosis of essential tremor (ET) or Parkinsonian (PD) tremor, visual inspection is not a reliable way to decompose the tremor. Tremor is multi-joint, with complexities and variability that need to be objectively characterized. Objective tools that help in understanding the multi-joint, multi-directional complexity and variability of tremor have not been developed.

Treatment of tremor involves pharmacological and surgical interventions. In patients with essential tremor, two agents, primidone and propranolol, have proven efficacy. ${ }^{2}$ In addition, for those presenting with severe disability, deep brain stimulation (DBS) of the thalamus (ventrointermediate nucleus (VIM nucleus)) as well as thalamotomy have been considered. ${ }^{3}$ However, substantial long-term side effects and risks exist and, as a result, many patients do not seek out help and remain untreated.

In patients with Parkinson disease, symptomatic treatment remains predominantly in the dopaminergic domain. Although

From the London Health Sciences Centre ( FR, CB, MJ); Department of Neurology (DD,MJ); Department of Health Rehabilitation Sciences (AR-S); Department of Physiology and Pharmacology (PB); Western University, London, ON, Canada; Department of Electrical Engineering (FR), University of Bonab, Velayat Highway, Bonab, Iran. Received June 13, 2014. Final Revisions Submitted December 19, 2014. Correspondence to: Fariborz Rahimi, Department of Clinical Neurological Sciences, Lawson Health Research Institute, 339 Windermere Road, A10-026, London, ON, Canada N6A 5A5. Email: frahimi@ bonabu.ac.ir. 
non-motor symptoms of PD such as postural instability, swallowing difficulty and mood are found to be less responsive to this form of treatment, most cardinal motor symptoms that represent PD respond very well to dopaminergic replacement. However, as the pre-eminent hyperkinetic motor symptom of PD, tremor remains difficult to treat. ${ }^{2,4-7}$ Data on the benefit of medications such as anticholinergics. ${ }^{5,8-9}$ levodopa, ${ }^{9}$ ) and dopamine agonists. ${ }^{2,4,9}$ for the treatment of tremor are inconsistent, and especially in the case of the agonists, retrospective. Anticholinergics produce substantial side effects and are contraindicated in many patients. ${ }^{8,9}$ The need for an effective and well tolerated treatment of tremor therefore remains a significant unmet need in PD.

The use of botulinum toxin (BoNT A) for the treatment of focal dystonia is well established. ${ }^{10}$ While injection of BoNT A in the extremities is a viable option for the treatment of tremor, ${ }^{7,11,12}$ it has not been indicated or widely adopted for clinical treatment, given impairment of limb function from excessive or unwanted muscle weakness. ${ }^{6}$ As with all injections in the limbs, especially in the upper limbs, injectors face difficulties for injection including identification of the dynamics of the movement (movement characterization), appropriate muscle selection due to multiple muscles contributing to the same movement direction, dosing per muscle per injection site, and muscle localization for injection. Clinical experience and extensive anatomical knowledge do provide insight into the issues related to dosing per muscle and the primary actions of muscles. Similarly, muscle localization for injection can be achieved using targeting technologies such as electromyography (EMG) or ultrasound. Accurate movement characterization is required for optimizing muscle selection. However, prior to all of this, the first step, of characterization of the dynamics of the movement at the joint and therefore the decomposition of the tremor at the joint, needs to be done. The clinician selects muscles to inject entirely on this decomposition of the dynamics of the joint movement. Currently, such characterization is done entirely on the basis of visual inspection and "gestalt" of the injector. Due to the number of factors involved, such as the variability of the tremor and differences across patients, visually-guided assessments and subsequent injections are prone to failure and side effects, ${ }^{5,10}$ limiting the use of BoNT A for tremor treatment.

Kinematic methodology is well established for studying the dynamics of movement in the upper limb. ${ }^{13,14}$ Technological advances have made this a reliable and viable option in the characterization of complex movements such as tremor. Wrist tremor is variable and has three anatomical components of movement: flexion/extension (F/E), radial/ulnar $(\mathrm{R} / \mathrm{U})$, pronation/ supination $(\mathrm{P} / \mathrm{S})$. Hence, visually-guided judgment of the complexity of movement over time may be difficult. Kinematic methodology can allow for objective assessment of all these variables, potentially leading to improved characterization of tremor dynamics.

In order to understand the biomechanics of tremor in both ET and $\mathrm{PD}$, this study aimed to evaluate the composition (how much $\mathrm{F} / \mathrm{E}, \mathrm{R} / \mathrm{U}, \mathrm{P} / \mathrm{S}$ ) of these tremor types at the wrist and to demonstrate the complexity of dynamics of the tremor. Since the dynamics of motion are what the clinician uses to determine which muscles they might inject, an important second goal of the study was to study if the clinician would make a different selection of muscles based on their visual assessment as compared with the data provided by kinematics. These two methods of muscle selection were, however, not compared in regards to efficacy after BoNT A injections but simply to highlight the difficulties associated with tremor dynamics. Finally, based on kinematics, this paper offers a general framework of tremor dynamics that could be considered for clinical injection protocols in the absence of kinematics.

\section{MeTHODS}

A sample of ET and PD patients was recruited for participation in the study by a single Movement Disorders Neurologist (MJ) from a tertiary care movement disorders clinic. Patients were enrolled in a larger ongoing study on the optimization of BoNT A injection for focal hand tremor over an eight month period. Baseline data for the first 11 patients with clinically confirmed ET and 17 with PD were studied (Table 1). The diagnosis of ET by a movement disorders specialist (MJ) was based on the current standards. ${ }^{15,16}$ All PD patients met the United Kingdom Brain Bank criteria for PD. Inclusion criteria involved all subjects to be 1) on stable medication management a minimum of six months prior to enrolment, with none withheld for this study 2) having tremor as their primary and most bothersome symptom 3) botulinum toxin naive. None of the subjects had other neurological disorders. For data recording, the motor dominant hand was considered for the ET patients. In PD patients, the hand reported to have the larger tremor amplitude was assessed, regardless of handedness, and all kinematic assessments were carried out in the "on" medication state. The study protocol was approved by the Human Subjects Research Ethics Board (HSREB) of Western University (REB\#17551) and all participants signed informed consent prior to their participation.

\section{Kinematic Recording Method}

Kinematic devices were used to record the composition of wrist tremor. Decomposition of tremor implies a breakdown into the main subcomponents of the tremor directions: flexion, extension, pronation, supination, radial, and ulnar deviation. Wrist F/E and $\mathrm{R} / \mathrm{U}$ were measured using a twin flexible axis electrogoniometer (SG65, Biometrics Ltd) placed across the wrist joint. Forearm P/S was measured using a 2D inclinometer (Noraxon®) secured to the dorsal surface of the hand. Together, sensors provided three degrees of freedom (DOF) angular measurements at the wrist. Finger tremor was also recorded using a linear accelerometer (three dimensional (3D), $6 \mathrm{~g}$, Noraxon ${ }^{\circledR}$ ) at the distal interphalangeal joint of the middle finger (Figure 1) giving three degrees of linear acceleration.

This measure provided us with an overall measure of tremor severity. The sensors were attached to standard positions using medical grade tape, and were connected to a laptop through TeleMyo 2400 T G2 and PC interface. Data were digitally sampled (at $1500 \mathrm{~Hz}$, using MyoResearch XP Master Edition 1.08.09 software, Noraxon (®) and saved for off-line processing and analysis. All recordings were performed in the seated position.

The accelerometer was a secondary measure only to assess overall severity and its readings were not used for decomposition of tremor at the wrist. It is possible that in addition to the movements of the tremor itself, the limb may move due to gravity and this variation would also be recorded. This movement should be of very low frequency and should be largely removed during the band -pass filtering process. In addition, this should not have an 
Table 1: Subject Demographics for ET and PD

\begin{tabular}{|c|c|c|c|c|c|c|c|c|c|}
\hline \multicolumn{5}{|l|}{ ET } & \multicolumn{5}{|c|}{ PD } \\
\hline Subject \# & Age & Gender & Yrs with Tremor & Side & Subject \# & Age & Gender & Yrs with Tremor & Side \\
\hline 1 & 64 & $\mathrm{M}$ & 10 & $\mathrm{R}$ & 1 & 47 & $\mathrm{M}$ & 11 & $\mathrm{R}$ \\
\hline 2 & 70 & $\mathrm{~F}$ & 33 & $\mathrm{R}$ & 2 & 66 & M & 3 & $\mathrm{R}$ \\
\hline 3 & 74 & $F$ & 11 & $\mathrm{~L}$ & 3 & 55 & $\mathrm{M}$ & 1 & $\mathrm{R}$ \\
\hline 4 & 69 & $\mathrm{M}$ & 4 & $\mathrm{R}$ & 4 & 57 & $\mathrm{~F}$ & 6 & $\mathrm{~L}$ \\
\hline 5 & 75 & M & 60 & $\mathrm{R}$ & 5 & 71 & M & 5 & $\mathrm{R}$ \\
\hline 6 & 72 & $\mathrm{M}$ & 6 & $\mathrm{R}$ & 6 & 58 & $\mathrm{M}$ & 7 & $\mathrm{R}$ \\
\hline 7 & 66 & M & 7 & $\mathrm{R}$ & 7 & 60 & F & 6 & $\mathrm{R}$ \\
\hline 8 & 74 & M & 4 & $\mathrm{R}$ & 8 & 69 & M & 25 & $\mathrm{~L}$ \\
\hline 9 & 75 & $\mathrm{~F}$ & 50 & $\mathrm{R}$ & 9 & 67 & F & 5 & $\mathrm{~L}$ \\
\hline 10 & 80 & $\mathrm{~F}$ & 3 & $\mathrm{R}$ & 10 & 63 & $\mathrm{M}$ & 6 & $\mathrm{R}$ \\
\hline \multirow[t]{7}{*}{11} & 47 & M & 20 & $\mathrm{~L}$ & 11 & 62 & M & 4 & $\mathrm{R}$ \\
\hline & & & & & 12 & 80 & $\mathrm{M}$ & 1 & $\mathrm{R}$ \\
\hline & & & & & 13 & 74 & M & 9 & $\mathrm{~L}$ \\
\hline & & & & & 14 & 72 & $\mathrm{M}$ & 7 & $\mathrm{~L}$ \\
\hline & & & & & 15 & 60 & $\mathrm{M}$ & 4 & $\mathrm{R}$ \\
\hline & & & & & 16 & 67 & $\mathrm{M}$ & 6 & $\mathrm{~L}$ \\
\hline & & & & & 17 & 67 & $\mathrm{~F}$ & 5 & $\mathrm{~L}$ \\
\hline Total & $69.6 \pm 8.8$ & $7 \mathrm{M}$ & $18.9 \pm 20.0$ & $9 \mathrm{R}$ & & $64.4 \pm 8.0$ & $13 \mathrm{M}$ & $6.5 \pm 5.4$ & $10 \mathrm{R}$ \\
\hline
\end{tabular}

$\mathrm{ET}=$ essential tremor, $\mathrm{PD}=$ Parkinspn's Disease, Yrs = years

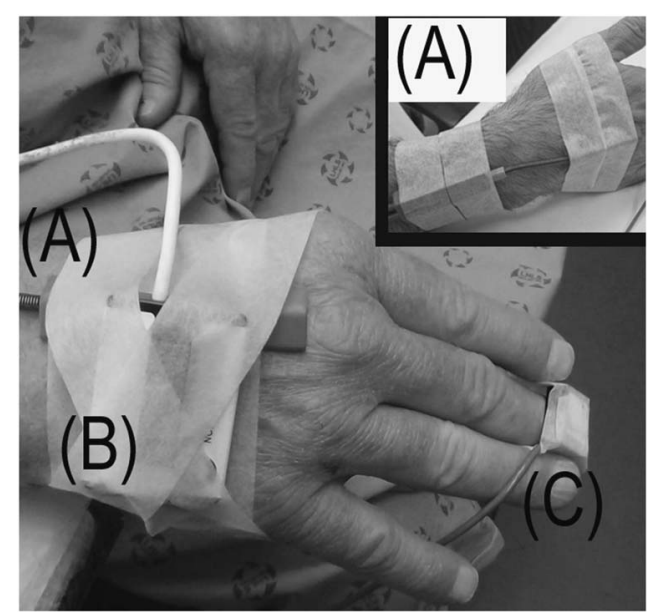

Figure 1: Tremor was measured by angle at the wrist, and by acceleration at the interphalangeal joint. A) An electro-goniometer measured wrist $F / E$ and $R / U$. B) An inclinometer measured forearm $P / S . C)$ A light-weight $3 D$ accelerometer collected distal finger movements as a measure of overall tremor severity.

impact on the angular values measured at the wrist which are used for the decomposition.

After attaching the sensors, the hand was placed against a fixed vertical plane in neutral $\mathrm{P} / \mathrm{S}$, neutral R/U deviation, and neutral F/E. Five seconds of data in this neutral position at rest (rest-neut) was used for calibration. Subjects then performed a series of five tasks: rest, posture, posture-neutral (posture-neut), loaded hand in posture, and finger-to-nose kinesis, each ten seconds in duration.
This series was repeated a total of three times. To avoid possible effects of external loads and larger voluntary movements on wrist tremor composition, only the simpler tasks/positions were considered. Therefore, only the two tasks of rest and posture that are classically assessed in clinical neurological exam to elucidate tremor were used in composition analysis. Resting tremor was assessed with hands relaxed in neutral pronation on the subject's lap, and postural tremor was assessed with shoulders flexed at 90 degrees, arms extended anteriorly and pronated. Directional bias was studied in the pronated position for R/U and in posture-neut position for F/E and P/S. Start time for every task in a trial was recorded for signal processing.

\section{Kinematic Data Processing}

Signal processing was performed in MatLab ${ }^{\circledR}$ (MathWorks, R2011a). For each subject data file, the segments corresponding to each trial were extracted for every task. Each segment included three angular position signals for the wrist, and three linear acceleration signals for the finger. For each angular position signal, the mean value during neutral position calibration was subtracted before further processing. All tremor signals (both angular position and acceleration) were band-pass filtered $(2-20 \mathrm{~Hz}$, leastsquared finite impulse response filter, order 2000). Signals were symmetrically padded on both ends. For each tremor signal, after filtering, root-mean-squared (RMS) value was calculated as the measure of amplitude to avoid filter transient effects. Amplitude for 3D finger tremor, amplitude for 3-components of wrist tremor, and directional bias of each component during trials were calculated for 3-trials of rest and for 3-trials of posture. Three 
dimensions of linear acceleration at the finger were combined (RMS) to provide overall tremor severity. Percent contribution for each of the three components to wrist tremor was determined with respect to the summed $3 \mathrm{D}$ angular amplitude $(\mathrm{F} / \mathrm{E}+\mathrm{R} / \mathrm{U}+\mathrm{P} / \mathrm{S})$. Directional bias for each of the 3-components were calculated by averaging the signal, taking into account direction (positive $=F / R / P$; negative $=\mathrm{E} / \mathrm{U} / \mathrm{S}$ ). Only the angular data for wrist movement was used for tremor decomposition at the wrist. The acceleration data was used for generating an overall tremor amplitude of the limb so as to reflect how the Unified Parkinson's Disease Rating Scale (UPDRS) items are measured.

\section{Non-Kinematic Measures Method (Clinical Scales)}

The following clinical scales of tremor were available for $8 \mathrm{ET}$ and $11 \mathrm{PD}$ patients. A single assessor (MJ) conducted the administration of UPDRS for the assessed hand. Items 20 (rest tremor: hands L/R) and 21 (hands action tremor: L/R) were collected for all patients. In the same data collection session, subjects were asked to draw the Archimedes spiral and a straight line as part of Fahn-Tolosa-Marin tremor rating scale for both hands. ${ }^{17}$ Tremor scores in lines and spirals drawing ranged from 0-4, and were evaluated by a separate assessor for all patients.

\section{Agreement between Kinematic and Clinical Derived Muscle Selection Schemes}

For the same group of subjects with recorded clinical scales (8 ET and $11 \mathrm{PD}$ patients), effect of tremor evaluation method on the choice of muscles selected for potential injection was examined. The clinical assessment was based on visual observation and the scores of the clinical scales used. The clinician
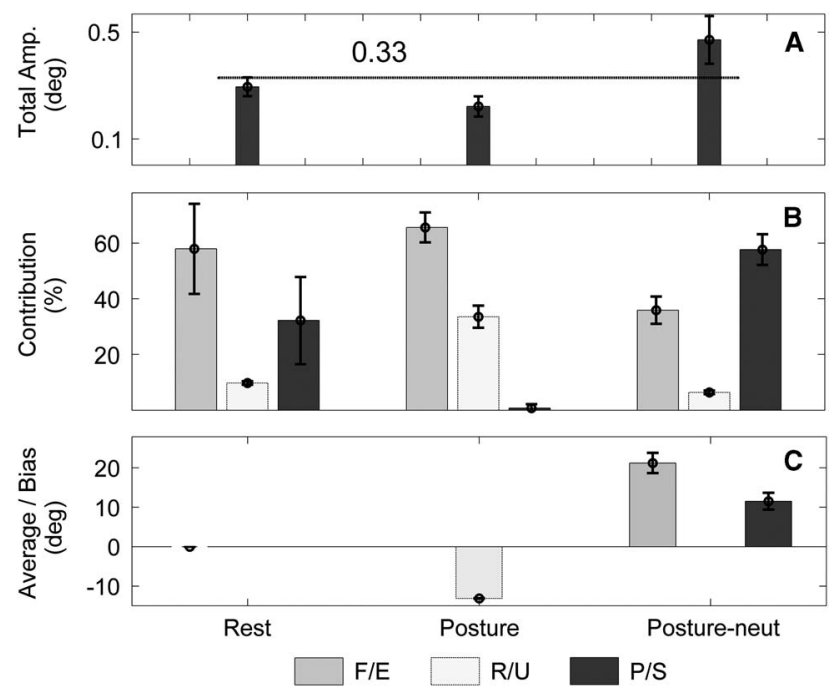

Figure 2: Amplitude, composition, and directional bias of tremor at the wrist for PD subject \#9. A) Root mean square combined amplitude of the 3-DOF in wrist tremor for rest, posture and neutral posture, with mean and standard deviations of the amplitude over three trials are presented. The grand average (horizontal line) is also presented. B) The contribution of each component $(F / E, R / U$, and $P / S)$ to the wrist tremor for each posture. $C$ ) The directional bias in each group of antagonist muscles (DOF). For P/S and F/E such a situation would be posture in neutral pronation (posture-neut). The figure shows that this tremor is predominantly a $F / E$ and $P / S$ type tremor at rest with bias towards flexion and pronation. selected the muscle groups for injection and the dosages that may be required for BoNT A injections (scheme 1). After recruitment of all subjects, kinematic analysis data was presented in a randomized order to the same clinician who was blinded to the clinical assessment of the patients. Kinematic data gave the direction of the movement, the amplitude and the relative contributions of each tremor component without any identifiers (See Figure 2).

These pairs included P/S at the forearm, F/E and R/U at the wrist. Similar to the visually-based clinical determination, the clinician then selected injection parameters, including the muscles and the possible dosage of BoNT A, for optimized outcome (scheme 2).

A comparison was made between the clinically (scheme 1) versus kinematically (scheme 2) determined muscles selected for possible injection, and an agreement percentage was calculated. For each subject, a muscle present in either selection method was assigned an agreement value. A muscle selected in both schemes was assigned the value 1 (agreement) while a muscle selected only in one scheme was assigned 0 (disagreement). For each group of subjects, mean agreement was calculated for each muscle as:

Agreement $(\%)=\frac{\# \text { agreement }}{\# \text { agreement }+\# \text { disagreement }} \times 100(\%)^{1}$

The mean agreement was reported per muscle. The agreement analysis did not involve comparison of injection dosage. Finally, overall agreement based on every single muscle selection was reported for each group of subjects along with the average number of muscles chosen for injection visually and kinematically

\section{Statistical Analyses}

Tremor acceleration amplitudes usually have skewed distributions and log-transformation is suggested in the literature. ${ }^{18-20}$ Therefore, overall finger tremor (combined 3D) amplitudes were log-transformed before analysis. The log-transformed data met criteria for parametric analysis. Average amplitude over three trials was compared in a two-way analysis of variance (ANOVA) between effects of diagnosis and repeated measures for rest and postural positions. Alpha level was set at.05 and Tukey's honest significance test was conducted for post-hoc analysis. The accelerometric data was not used for any of the decomposition analysis.

Percent contribution for each of the three components of wrist tremor was averaged over three trials. The data violated homogeneity of variances (Levene's test) as well as homogeneity of covariances (Box M test). Therefore, components' contributions were separately compared for ET and PD patients, at rest and in posture, using Kruskall-Wallis nonparametric test.

The averaged directional bias data over three trials, met criteria for parametric analysis. For each group of subjects, a separate univariate ANOVA compared directional bias in each of the wrist tremor components (F/E, R/U, P/S). Confidence intervals (95\%) were used to examine if the average bias for a component is significantly positive or negative. Statistical analyses were performed in STATISTICA® 8.0, StatSoft Inc.

\section{Results}

Eleven ET patients $(70 \pm 8.8$ years $)$ and 17 PD patients $(64 \pm 8.0$ years $)$ were assessed with the demographics summarized in Table 1. 
Table 2: Clinical and Kinematic Tremor Scores.

\begin{tabular}{|c|c|c|c|c|c|c|c|c|}
\hline \multicolumn{9}{|l|}{ ET } \\
\hline $\begin{array}{l}\text { Subject } \\
\#\end{array}$ & $\begin{array}{l}\text { Finger Accel. } \\
\text { (g) Rest }\end{array}$ & $\begin{array}{l}\text { Finger Accel. (g) } \\
\text { Posture }\end{array}$ & $\begin{array}{l}\text { Wrist Angle } \\
\text { (deg) Rest }\end{array}$ & $\begin{array}{l}\text { Wrist Angle (deg) } \\
\text { Posture }\end{array}$ & $\begin{array}{l}\text { UPDRS Item } 20 \text { score } \\
\text { Hand only }\end{array}$ & $\begin{array}{l}\text { UPDRS Item } \\
21 \text { Score }\end{array}$ & $\begin{array}{c}\text { Spiral } \\
\text { Drawing score }\end{array}$ & $\begin{array}{c}\text { Line } \\
\text { Drawing } \\
\text { score }\end{array}$ \\
\hline 1 & 1.08 & 0.55 & 0.42 & 0.25 & 2 & 2 & 2 & 2 \\
\hline 2 & 0.14 & 0.29 & 0.05 & 0.24 & 0 & 3 & 3 & 3 \\
\hline 3 & 0.37 & 3.13 & 0.30 & 2.51 & 2 & 3 & 4 & 4 \\
\hline 4 & 0.12 & 0.67 & 0.09 & 0.87 & 0 & 2.5 & 2 & 2 \\
\hline 5 & 0.08 & 0.09 & 0.04 & 0.06 & 1 & 2.5 & 4 & 4 \\
\hline 6 & 0.01 & 0.11 & 0.15 & 0.71 & 0 & 3 & 2 & 2 \\
\hline 7 & 0.14 & 0.62 & 0.69 & 2.31 & 0 & 3 & 1 & 1 \\
\hline 8 & 0.02 & 0.02 & 0.16 & 0.17 & 0 & 2 & 2 & 1 \\
\hline 9 & 0.09 & 0.16 & 0.08 & 0.13 & & & & \\
\hline 10 & 0.05 & 0.17 & 0.06 & 0.19 & & & & \\
\hline \multirow[t]{2}{*}{11} & 0.11 & 0.30 & 0.19 & 0.29 & & & & \\
\hline & $0.2 \pm 0.3$ & $0.6 \pm 0.9$ & $0.2 \pm 0.2$ & $0.7 \pm 0.9$ & $0.6 \pm 0.9$ & $2.6 \pm 0.4$ & $2.5 \pm 1.1$ & $2.4 \pm 1.2$ \\
\hline \multicolumn{9}{|l|}{ PD } \\
\hline 1 & 4.99 & 6.97 & 2.09 & 6.19 & 3.5 & 3 & 3 & 2 \\
\hline 2 & 1.02 & 3.17 & 0.28 & 0.62 & 2.5 & 2.5 & 0 & 0 \\
\hline 3 & 0.08 & 0.14 & 0.04 & 0.10 & 3 & 0 & 0 & 0 \\
\hline 4 & 3.91 & 5.21 & 1.35 & 2.79 & 3 & 2.5 & 4 & 4 \\
\hline 5 & 0.40 & 0.34 & 0.24 & 0.15 & 2 & 1 & 1 & 1 \\
\hline 6 & 4.03 & 5.59 & 2.51 & 2.47 & 3.5 & 3 & 1 & 0 \\
\hline 7 & 0.11 & 0.30 & 0.07 & 0.15 & 3 & 1 & 1 & 1 \\
\hline 8 & 2.53 & 5.76 & 0.49 & 1.40 & 2.5 & 2 & 4 & 4 \\
\hline 9 & 0.33 & 0.26 & 0.30 & 0.18 & 1.5 & 1 & 1 & 1 \\
\hline 10 & 0.28 & 0.23 & 0.20 & 0.19 & 2 & 0 & 0 & 0 \\
\hline 11 & 0.06 & 0.10 & 1.98 & 0.08 & 3 & 0 & 1 & 0 \\
\hline 12 & 0.19 & 0.23 & 0.14 & 0.12 & & & & \\
\hline 13 & 0.08 & 0.15 & 0.05 & 0.08 & & & & \\
\hline 14 & 0.37 & 0.22 & 0.32 & 0.12 & & & & \\
\hline 15 & 1.11 & 1.95 & 0.74 & 0.74 & & & & \\
\hline 16 & 0.21 & 0.73 & 0.10 & 0.35 & & & & \\
\hline 17 & 6.61 & 7.01 & 4.30 & 4.82 & & & & \\
\hline Avg,SD & $1.5 \pm 2.1$ & $2.3 \pm 2.7$ & $0.9 \pm 1.2$ & $1.2 \pm 1.8$ & $2.7 \pm 0.6$ & $1.5 \pm 1.2$ & $1.5 \pm 1.5$ & $1.2 \pm 1.5$ \\
\hline
\end{tabular}

Clinical data was not available for ET subjects 9-11 for ET and PD subject 12-17.

$\mathrm{ET}=$ essential tremor, $\mathrm{PD}=$ Parkinsonian tremor, accel $=$ acceleration tremor, angle $=$ angular tremor $(\mathrm{deg})=\mathrm{degree}, \mathrm{Avg}=\mathrm{average}, \mathrm{SD}=\mathrm{standard}$ deviation.

The summary of tremor scores is presented in Table 2. Average finger tremor (acceleration, before log-transformation) and wrist tremor (angle) amplitudes over all rest and posture trials are also presented for each subject. Summed Items 20 (only hand) and 21 from UPDRS are presented for each subject along with the scores in line and spiral drawings.

Since the 3D accelerometric measurement at the finger would show tremor originating from the fingers, wrist and elbow, we used the finger tremor amplitude to represent overall tremor severity. Tremor amplitude of ET at rest was significantly lower $(F(1,26)=5.25, p=.030$, and post-hoc Tukey's HSD test $)$ than
ET at posture and PD at rest, while PD at rest and posture were not significantly different. In addition, ET and PD at posture were also not significantly different. These data are presented in Figure 3-A.

In order to compare overall tremor severity between kinematic and clinical measures (UPDRS tremor score), acceleration amplitudes at rest and posture for finger tremor were averaged over three trials. Wrist angle was also averaged in the same way. These two measures were then individually compared to the summed Items 20 and 21 of the UPDRS, which served as a clinical indicator of overall tremor. Since the finger movement was recorded as an acceleration and wrist movement as an angle, these 

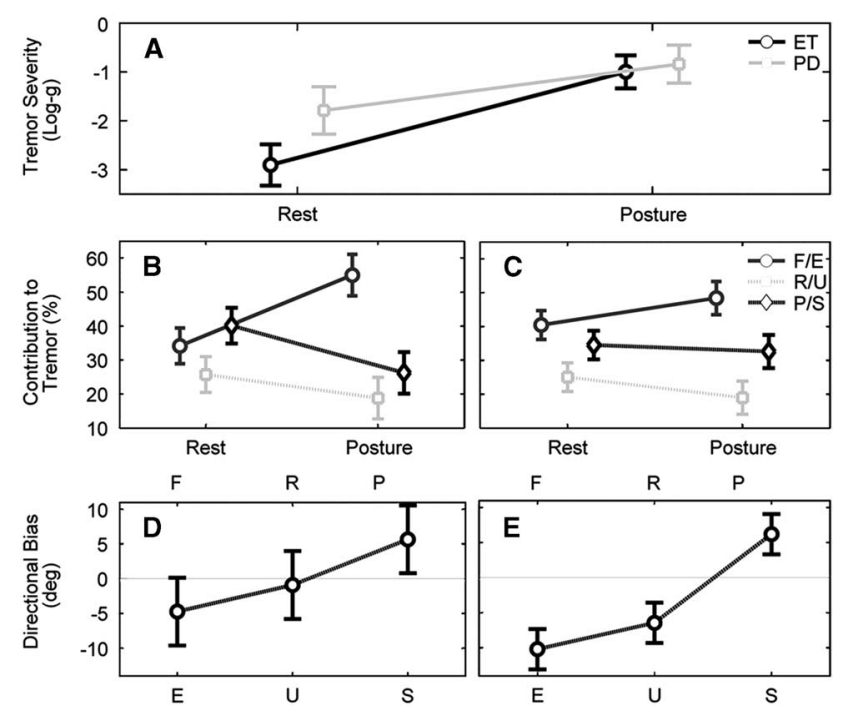

Figure 3: A) Finger amplitude for overall tremor severity for ET and $P D$ averaged over three trials of each condition. B) Composition of wrist tremor contrasted between rest and posture tasks for ET and C) PD. Contribution was calculated for each DOF $(F / E, R / U$, and $P / S)$ amplitude with respect to the sum of all 3-DOF amplitudes. D) Directional bias across 3-DOF in wrist tremor for ET and E) PD. Confidence interval outside zero is considered significant bias.

could not be summed. There was a strong linear dependence between UPDRS Items $(20+21)$ and the kinematic measures of tremor amplitude in both ET and PD (Pearson's correlation coefficient, $r=0.84, r=0.84$ for log-transformed average finger tremor, and for average angular wrist tremor amplitudes respectively). There was no significant difference in summed UPDRS scores of hand rest and postural tremors between ET patients $(95 \% \mathrm{CI}[2.6,3.9])$ and $\mathrm{PD}(95 \% \mathrm{CI}[3.2,5.0])$ implying that there was no difference in the two groups for tremor severity. However, separated UPDRS Item 20 (hand tremor at rest: ET: [0, 1.3], PD: [2.3, 3.1]) and 21 (action tremor: ET: [2.3, 2.9], PD: [0.7, 2.2]) were significantly different between the two groups of patients. Similarly, the kinematic measures at rest and posture in ET and PD showed no significant difference (finger rest: ET: [0.0, 0.5], PD: [0.5, 2.7]; finger posture: ET: [0.0, 1.4], PD: [0.9, 4.2]; wrist rest: ET: [0.1, 0.4], PD: [0.3, 1.4]; wrist posture: ET: [0.2, 1.6], PD: $[0.2,2.4])$. No significant differences were found in line drawing (ET: [1.8, 3.2], PD: [0.6, 2.3]) or spiral drawing (ET: $[1.6,3.1]$, PD: $[0.3,2.0])$ scores.

The composition of tremor, for both groups of subjects and for both tasks of rest and posture, is presented in Figure 3-b and c. For ET at rest, none of the components was found to dominate the wrist tremor (Kruskal-Wallis test: $H(2, N=33)=3.76, p=.153$ ). For ET in posture, F/E was found to dominate the other two components $(H=12.26, p=.002)$. For PD both at rest and in posture, F/E was significantly larger than $\mathrm{R} / \mathrm{U}(H(2, N=51)$ $=6.28, p=.043 ; H=12.78, p=.002$ respectively), but not $\mathrm{P} / \mathrm{S}$.

In order to divide each degree of freedom separately, we calculated the directional bias for each pair of antagonist muscles at the wrist (F vs. E, R vs. U, P vs. S) and not at the finger. This indicated whether one directional component dominated for both ET and PD. The average directional bias for each of the three wrist tremor components and for both groups of subjects is presented in Figure 3-d and e. For both groups of subjects, directional bias

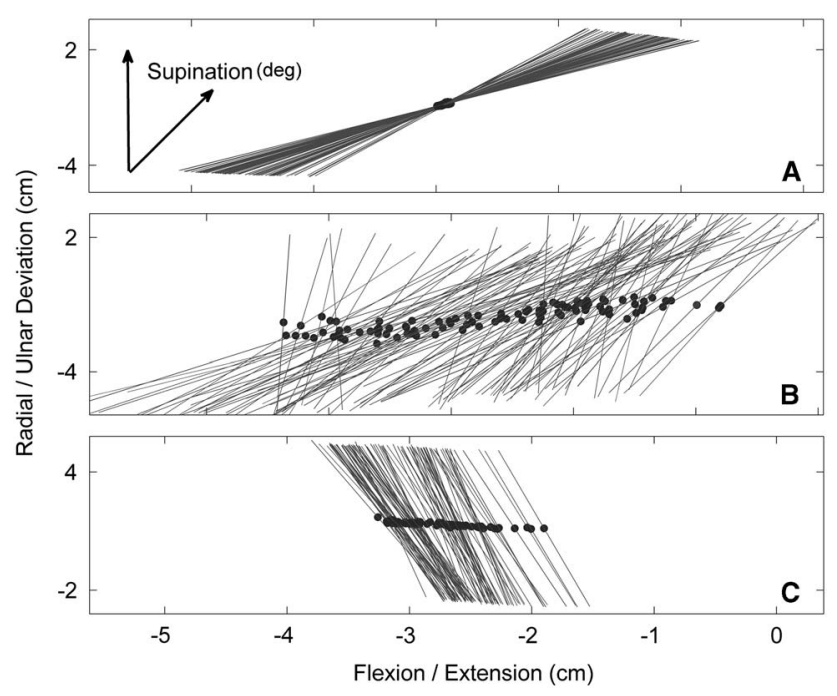

Figure 4: Illustration of wrist tremor complexity in 3 DOF for three subjects. Each line represents the motion of the wrist recorded every $0.1 \mathrm{sec}$. Movement of the red dot along the $X$-axis represents $F / E$, along the $Y$-axis $R / U$, and the line rotation (angle) representing $P / S$. In A) for $P D$ subject \#6 at rest, the tremor is predominantly P/S with minimal $F / E$ or $R / U$ deviations. In B) for PD subject \#2 in posture, the tremor is a combination of F/E and P/S. In C) for ET subject \#10 in posture, the tremor is predominantly $F / E$ with slight $P / S$.

was significantly different among the components (ET, $F(2$, $30)=4.84, p=.015 ;$ PD $, F(2,48)=36.18, p<.001)$. For ET patients, the only significant average bias was for $\mathrm{P} / \mathrm{S}$ which was toward pronation. For PD patients, all three components had significant average directional biases. The bias for $\mathrm{F} / \mathrm{E}$ was toward extension, for $\mathrm{R} / \mathrm{U}$ toward ulnar deviation, and for $\mathrm{P} / \mathrm{S}$ toward pronation.

With respect to composition, wrist tremor movements were often complex with none of the components (F/E, R/U and P/S) clearly dominating the tremulous motion. To assess this complexity, a component was deemed to be dominant if contribution was $>70 \%$ (arbitrarily). For each subject, rest and posture trials were separately averaged for ET and PD, and then evaluated for the occurrence of any component above this threshold. This analysis revealed that for ET the dominance percentages were (rest: $0 \%$, posture: $36 \%$ ) and for PD (rest: $23 \%$, posture: $23 \%$ ). As an example, wrist tremor composition for three different subjects is demonstrated in Figure 4. This example clearly demonstrates that visual decomposition of the wrist tremor movement, depending on the patient and situation, could vary from simple (case-a) to very challenging (case-b).

Agreement between scheme 1 and scheme 2 in selecting muscles that contribute to tremor was then evaluated. When a specific muscle appeared in both the schemes, an agreement number of 1 was assigned; if the muscle appeared in only one of the two schemes, the number was 0 . The determination was done for every muscle that was used in the schemes and the list is presented, with the agreements in Table 3.

\section{Discussion}

Tremor is a relatively treatment-resistant symptom of PD, and ET is one of the most common movement disorders. ${ }^{21}$ This study 
Table 3: Agreement in all muscles selected for injection.

\begin{tabular}{|c|c|c|c|c|c|}
\hline \multicolumn{2}{|l|}{ Muscle } & \multicolumn{2}{|c|}{ ET } & \multicolumn{2}{|c|}{ PD } \\
\hline Name & Abr. & Present in a selection & Agreem-ent (\%) & Present in a selection & Agreem-ent (\%) \\
\hline Flexor carpi ulnaris & FCU & 8 & 50 & 11 & 64 \\
\hline Flexor carpi radialis & FCR & 7 & 57 & 10 & 70 \\
\hline Extensor carpi ulnaris & $\mathrm{ECU}$ & 7 & 29 & 8 & 75 \\
\hline Extensor carpi radialis & ECR & 7 & 29 & 8 & 63 \\
\hline Supinator & SUP & 6 & 33 & 8 & 50 \\
\hline Pronator teres & PRT & 6 & 33 & 7 & 57 \\
\hline Pronator quadratus & PRQ & 5 & 40 & 6 & 17 \\
\hline Biceps brachii & BIC & 4 & 0 & 5 & 20 \\
\hline Triceps brachii & TRI & 0 & 0 & 1 & 0 \\
\hline Flexor digitorum superficialis & FDS & 0 & 0 & 1 & 0 \\
\hline Flexor pollicis longus & FPL & 0 & 0 & 1 & 0 \\
\hline Overall Agreement & & & $36 \%$ & & $53 \%$ \\
\hline \# Muscles Selected Kinematically & & 4 & & 4 & \\
\hline \# Muscles Selected Clinically & & 5 & & 5 & \\
\hline
\end{tabular}

$\mathrm{ET}=$ essential tremor, $\mathrm{PD}=$ Parjkinsonian tremor.

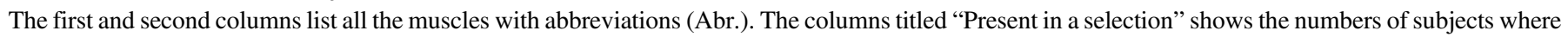
that particular muscle was chosen. The column titled "Agreement" shows the percentage agreement for that muscle between scheme 1 and scheme 2.

addresses three major assumptions: 1) that because tremor is easy to spot, it should be easy to treat 2) that because tremor is easy to visualize, tremor dynamics must be easy to decompose and 3) that ET tremor presents as an action tremor (postural or kinetic) while PD tremor presents as a pill-rolling rest tremor. Detailed kinematic assessment of both tremor types to decompose tremor dynamics suggest these assumptions may not be true, and provide insightful clinical implications for selecting such management options as BoNT A for tremor.

Although tremor in ET and PD can involve the head, face, and tongue, the most common site remains the limbs, particularly the upper limbs. Subsequent functional impairment is a result of tremor and this can be substantially disabling if the dominant arm is affected. $^{22,23}$ In addition, the presence of tremor is an obvious visible symptom which can be cosmetically disabling, making patients feel as though they "stand out" as patients. Due to such functional and cosmetic disability, an effective treatment method for focal wrist tremor remains an important need in affected individuals. While options exist for management of ET and PD tremor, the side effects of medication and risk in brain surgery pose considerable risk, especially in the older age group. ${ }^{3}$

Botulinum toxin injection therapy has shown efficacy and is indicated for the management of focal disorders such as torticollis, blepharospasm, writing tremor, task-specific writing dystonia, and upper limb spasticity. ${ }^{24-28}$ Although tremor has been treated with BoNT A, the studies have been open-label. ${ }^{29}$ or small and the results of BoNT A have not generally been particularly favorable. ${ }^{3,7,30}$ In the study by Jankovic et al. for ET, injection with BoNT A indeed reduced postural tremor amplitude as measured by accelerometry and clinical rating scales. ${ }^{6}$ However, all patients had some degree of weakness as a side effect, and functional disability and action tremor did not improve significantly.
It is possible that despite the reduction in tremor, weakness overshadowed the improvement and resulted in a lack of significant functional improvement seen. Nevertheless, the authors did suggest that chemodenervation with BoNT A was a viable option for treatment of ET. However, this has not been largely accepted as an important treatment option by clinicians, nor is it reimbursed by insurance companies.

The lack of functional improvement using BoNT A is the side effect profile that is produced by the injection. Injections can produce substantial weakness in the muscles due to its wellknown action. ${ }^{31,32}$ This weakness is in the muscles injected and also in the adjacent muscles due to the spread of the toxin. It is known that this weakness and spread is dose and volume dependent. ${ }^{33,34}$ However, the most significant determinant of this side effect may be the selection of the appropriate and most responsible muscles that contribute to the tremor seen and the dosage injected within the muscles. The most important component of muscle selection is the clinician's ability to determine the predominant direction of movement of the affected body part. This is true even for dystonia and spasticity, the two other syndromes where BoNT A is successfully used. In these conditions, the movement is generally fairly stereotyped and the predominant postures of the body parts affected can be visually assessed by the clinician. However, when tremor is superimposed on, for example,cervical dystonia, the assessment of the movement and the subsequent injection pattern determination becomes that much more difficult.

To date, the tremor of PD and ET have been assumed to have well established "clinical features": rest tremor in PD and postural and kinetic tremor in ET. Additionally, the predominant composition of these tremor types has been also assumed to be $\mathrm{F} / \mathrm{E}$, mainly present at the wrist. 7,11,29,33,35-37 Finally, despite the complexity of such tremors, the judgment of which muscles to 
inject and the dosage of BoNT A required is achieved purely on visual inspection. Tremor in the upper limb can be complex to assess visually simply because of the number of body parts involved. For example, the tremor is present often at the elbow, wrist and in fingers. In addition, each of these joints has many degrees of freedom in terms of movement. The wrist can flex and extend, and show ulnar and radial deviation, while at the same time the elbow may show pronation-supination. Such multidimensional motion is then summed in producing the actual tremor. The clinician has to visually decompose these components and then determine the relative contributions of each in order to estimate which muscle groups to select for injection. In most cases this is a very difficult task and may over- or under-estimate the movement subcomponents. If this happens, the injections of BoNT A may be given in incorrect muscle groups resulting in suboptimal benefit and increased side effects. Therefore, methods that assess tremor composition beyond simple accelerometric measurements become essential.

This study demonstrates that tremor, in both rest and posture, is present in ET and PD. In ET, the tremor is clearly posture predominant while in PD both rest and posture were equal in our cohort. The amplitude of PD tremor was overall higher in our subjects. In addition, significant variability existed in the tremor amplitude. These results are shown in panels A of Figure 2 and Figure 3. The postural component of PD tremor in our cohort may be a result of patients with more severe tremor than a typical PD patient but it emphasizes the point that tremor in posture can exist with as much severity in PD as rest and may contribute to functional disability doing tasks.

The complex composition of tremor in ET and PD is shown in Figure 3-b. In ET, at rest, all three components of F/E, R/U and P/ $\mathrm{S}$ are contributing almost equally. With posture, this composition changes significantly so that F/E becomes predominant. However, $\mathrm{P} / \mathrm{S}$ and $\mathrm{R} / \mathrm{U}$ do persist but at a much lower proportion. Hence, if a patient has predominant posture related issues with ET, the suggestion would be to begin injections with BoNT A with those muscle groups that contribute to flexion and extension. If the patient is also seen to have rest tremor and the diagnosis is still ET, then additional injections with $\mathrm{P} / \mathrm{S}$ and $\mathrm{R} / \mathrm{U}$ can be considered. Analysis of the directional bias of these components with respect to the contributions towards movement as seen in Figure 3-d show that the injections should be equally divided between the muscle groups contributing to $\mathrm{F} / \mathrm{E}$ and $\mathrm{R} / \mathrm{U}$ while pronators should receive more than supinators.

Figure 3-c also shows that in $\mathrm{PD}$ tremor $\mathrm{F} / \mathrm{E}$ and $\mathrm{P} / \mathrm{S}$ were equally significant at rest and in posture and contributed significantly higher than $\mathrm{R} / \mathrm{U}$ in both conditions. This suggests that when considering injections for PD tremor, both of these movement subcomponents should be injected from the start and probably in equal amounts. Analysis of directional bias of these components with respect to the contribution towards the tremor for these patients shows that the injection amounts should be divided between the antagonist muscles such that extensors should receive more than flexors; ulnar deviators more than radial deviators, and pronators more than supinators. One can hypothesize a decision tree where, extensor carpi radialis (ECR) and extensor carpi ulnaris ECU are injected, with ECU $>$ ECR while pronator quadratis(PRQ) and pronator teres (PRT) are injected more than supinator (SUP) and potentially biceps brachii (BIC). Since this study was undertaken to show the complexity and dynamics of the tremor, we have not specifically indicated the dosages of BoNT A to be used for injection but have shown the important issues that may guide injections. In addition, experienced injectors along with those that do not have access to kinematics may use their clinical judgment for the dosing, but with the knowledge as to which groups to target as they commence and continue their injections.

This study showed significant variability in the tremor parameters within (Figure 2) and between (Figure 3) subject groups. It has been already shown that, after tremor amplitude, the second most variable factor in wrist tremor is its composition. ${ }^{38}$ Furthermore, change in the task might considerably change the composition of wrist tremor (Figure 2). This means that a single and simple visual inspection of tremor in one situation might not be enough. In a clinical setting it is extremely difficult to observe a patient's tremor in a variety of different positions and determine the overall composition of the tremor. Indeed, the clinician does not have the ability to summate over a period of time or over different positions. Due to this variability and the difficulty in perceiving this by simple visual inspection, it is quite likely that the muscle selection may not be optimal. Kinematics provide a reliable and reproducible, objective measure that solves in our mind, this very important issue of tremor decomposition.

Since the determination of the predominant characteristics of motion in the tremor is done visually, we compared the composition and the subsequent muscle selection for injection done by this method to what was provided by the kinematic assessment. Although this comparison was not the main goal of this study, low agreement between clinical assessment in the hands of an experienced injector and what was given by the objective kinematic assessment highlights the inherent difference between subjective visual and objective kinematic assessment of complexity of tremor. Table 3 shows that overall there was only a 36\% and 53\% agreement for muscles chosen visually versus in the blinded kinematic assessment, for ET and PD respectively. We do not know that the kinematic assessment was the more accurate one in terms of outcome after BoNT A injection. This would require a head-to-head comparative design which is a complex and large endeavour. The goal of this study was to reveal the dynamics of tremor and highlight the fact that the mediocre results from other studies of BoNT A injections based on visual assessment may at least in part be a result of a simplistic assessment of tremor. It makes logical sense that understanding the dynamics and then guiding the injections based upon kinematics may improve the clinical outcome of BoNT A treatment. This study is currently on-going at our centre.

\section{ACKNOWLEDGMENTS}

The authors thank the patients at the Movement Disorders Clinic, London Health Sciences Centre, London, Ontario, Canada. The study has partial industry support, by a pilot research grant, from Merz Pharma Canada.

\section{DisCLOSURES}

The present study was partially funded by a pilot research grant from Merz Pharma Canada.

FR has the following disclosures: Teva Canada, Consultation, Consulting fees.

MJ has the following disclosures: Merz, Consulting, Consulting fee; Allergan, Advisor, Honoraria; Abbvie, Research support, 
Honoraria; CIHR, Researcher, Research support; MDDT ING, Director, Stocks. $\mathrm{DD}, \mathrm{AR}-\mathrm{S}, \mathrm{CB}$, and $\mathrm{PB}$ do not have anything to disclose.

\section{REFERENCES}

1. Louis ED, Ferreira JJ. How common is the most common adult movement disorder? Update on the worldwide prevalence of essential tremor. Mov Disord. 2010;25:534-41.

2. Wasielewski PG, Burns JM, Koller WC. Pharmacologic treatment of tremor. Mov Disord. 1998;13 Suppl 3:90-100.

3. Deuschl G, Raethjen J, Hellriegel H, Elble R. Treatment of patients with essential tremor. Lancet Neurol. 2011;10:148-61.

4. Miyasaki JM, Martin W, Suchowersky O, Weiner WJ, Lang AE. Practice parameter: initiation of treatment for Parkinson's disease: an evidence-based review: report of the Quality Standards Subcommittee of the American Academy of Neurology. Neurology. 2002;58:11-7.

5. Fishman PS. Paradoxical aspects of parkinsonian tremor. Mov Disord. 2008;23:168-73.

6. Jankovic J, Schwartz K, Clemence W, Aswad A, Mordaunt J. A randomized, double-blind, placebo-controlled study to evaluate botulinum toxin type A in essential hand tremor. Mov Disord. 1996;11:250-6.

7. Simpson DM, Blitzer A, Brashear A, et al. Assessment: Botulinum neurotoxin for the treatment of movement disorders (an evidencebased review): report of the Therapeutics and Technology Assessment Subcommittee of the American Academy of Neurology. Neurology. 2008;70:1699-706.

8. Katzenschlager R, Sampaio C, Costa J, Lees A. Anticholinergics for symptomatic management of Parkinson's disease. Cochrane Database Syst Rev. 2003:CD003735.

9. Schadt CR, Duffis EI, Charles PD. Pharmacological treatment of disabling tremor. Expert Opin Pharmacother. 2005;6:419-28.

10. Das CP, Truong D, Hallett M. Treatment of focal hand dystonia. In: Truong D, Dressler D, Hallett M, editors. Manual of Botulinum Toxin Therapy. Cambridge: Cambridge University Press; 2009.

11. Sheffield JK, Jankovic J. Botulinum toxin in tic disorders and essential hand and head tremor. In: Truong D, Dressler D, Hallett M, editors. Manual of Botulinum Toxin Therapy. Cambridge: Cambridge University Press; 2009.

12. Rahimi F, Bee C, Debicki D, Roberts AC, Bapat P, Jog M. Effectiveness of BoNT A in Parkinson's disease upper limb tremor management. Can J Neurol Sci. 2013;40:663-9.

13. Supuk T, Bajd T, Kurillo G. Assessment of reach-to-grasp trajectories toward stationary objects. Clin Biomech. 2011;26:811-8.

14. Casellato C, Zorzi G, Pedrocchi A, Ferrigno G, Nardocci N. Reaching and writing movements: sensitive and reliable tools to measure genetic dystonia in children. J Child Neurol. 2011;26:822-9.

15. Benito-Leon J, Louis ED. Update on essential tremor. Minerva Med. 2011;102:417-40.

16. Deuschl G, Bain P, Brin M. Consensus statement of the Movement Disorder Society on Tremor. Ad Hoc Scientific Committee. Mov Disord. 1998;13 Suppl 3:2-23.

17. Fahn S, Tolosa E, Concepcion M. Clinical rating scale for tremor. In: Jankovic J, Tolosa E, editors. Parkinson's disease and movement disorders. Baltimore: Williams and Wilkins, 1993, p. 271-80.

18. Elble RJ, Pullman SL, Matsumoto JY, Raethjen J, Deuschl G, Tintner R. Tremor amplitude is logarithmically related to 4- and 5-point tremor rating scales. Brain. 2006;129(Pt 10):2660-6.
19. Letz R, Gerr F. Reliability of some tremor measurement outcome variables in field testing situations. Neurotoxicology. 2000;21: 737-742.

20. Heroux ME, Pari G, Norman KE. The effect of inertial loading on wrist kinetic tremor and rhythmic muscle activity in individuals with essential tremor. Clin Neurophysiol. 2011;122:1794-801.

21. Benito-Leon J. How common is essential tremor? Neuroepidemiology. 2009;32:215-6

22. Norman KE, D'Amboise SN, Pari G, Heroux ME. Tremor during movement correlates well with disability in people with essential tremor. Mov Disord. 2011;26:2088-94.

23. Heroux ME, Parisi SL, Larocerie-Salgado J, Norman KE. Upperextremity disability in essential tremor. Arch Phys Med Rehabil. 2006;87:661-70.

24. Batla A, Stamelou M, Bhatia KP. Treatment of focal dystonia. Curr Treat Options Neurol. 2012;14:213-29.

25. Rana AQ, Vaid HM. A review of primary writing tremor. Int $\mathbf{J}$ Neurosci. 2012;122:114-8.

26. Papapetropoulos S, Singer C. Treatment of primary writing tremor with botulinum toxin type a injections: report of a case series. Clin Neuropharmacol. 2006;29:364-7.

27. Sheean G. Restoring balance in focal limb dystonia with botulinum toxin. Disabil Rehabil. 2007;29:1778-88.

28. Sheean G, Lannin NA, Turner-Stokes L, Rawicki B, Snow BJ. Botulinum toxin assessment, intervention and after-care for upper limb hypertonicity in adults: international consensus statement. Eur J Neurol. 2010;17 Suppl 2:74-93.

29. Pacchetti C, Mancini F, Bulgheroni M, et al. Botulinum toxin treatment for functional disability induced by essential tremor. Neurol Sci. 2000;21:349-53.

30. Rawicki B, Sheean G, Fung VS, Goldsmith S, Morgan C, Novak I. Botulinum toxin assessment, intervention and aftercare for paediatric and adult niche indications including pain: international consensus statement. Eur J Neurol. 2010;17 Suppl 2: $122-34$

31. Naumann M, Albanese A, Heinen F, Molenaers G, Relja M. Safety and efficacy of botulinum toxin type A following long-term use. Eur J Neurol. 2006;13 Suppl 4:35-40.

32. Crowner BE, Torres-Russotto D, Carter AR, Racette BA. Systemic weakness after therapeutic injections of botulinum toxin a: a case series and review of the literature. Clin Neuropharmacol. 2010;33:243-7.

33. Brin MF, Lyons KE, Doucette $\mathrm{J}$, et al. A randomized, double masked, controlled trial of botulinum toxin type A in essential hand tremor. Neurology. 2001;56:1523-8.

34. Lim EC, Seet RC. Botulinum toxin: description of injection techniques and examination of controversies surrounding toxin diffusion. Acta Neurol Scand. 2008;117:73-84.

35. Popović Maneski L, Jorgovanović N, Ilić V, et al. Electrical stimulation for the suppression of pathological tremor. Med Biol Eng Comput. 2011;49:1187-93.

36. Raethjen J, Lindemann M, Schmaljohann H, Wenzelburger R, Pfister G, Deuschl G. Multiple oscillators are causing parkinsonian and essential tremor. Mov Disord. 2000;15:84-94.

37. Rozman J, Bartolic A, Ribaric S. A new method for selective measurement of joint movement in hand tremor in Parkinson's disease patients. J Med Eng Technol. 2007;31:305-11.

38. Rahimi F, Bee C, South A, Debicki D, Jog M. Variability of hand tremor in rest and in posture-a pilot study. Conf Proc IEEE Eng Med Biol Soc. 2011;2011:470-3. 